ARTICLE

Received 11 Nov 2013 | Accepted 14 Aug 2014 | Published 26 Sep $2014 \quad$ DOl: 10.1038/ncomms5997

\title{
Is wave-particle objectivity compatible with determinism and locality?
}

Radu lonicioiu ${ }^{1,2}$, Thomas Jennewein ${ }^{3,4}$, Robert B. Mann ${ }^{3,4,5} \&$ Daniel R. Terno ${ }^{6}$

Wave-particle duality, superposition and entanglement are among the most counterintuitive features of quantum theory. Their clash with our classical expectations motivated hidden-variable (HV) theories. With the emergence of quantum technologies, we can test experimentally the predictions of quantum theory versus $\mathrm{HV}$ theories and put strong restrictions on their key assumptions. Here, we study an entanglement-assisted version of the quantum delayed-choice experiment and show that the extension of $\mathrm{HV}$ to the controlling devices only exacerbates the contradiction. We compare HV theories that satisfy the conditions of objectivity (a property of photons being either particles or waves, but not both), determinism and local independence of hidden variables with quantum mechanics. Any two of the above conditions are compatible with it. The conflict becomes manifest when all three conditions are imposed and persists for any non-zero value of entanglement. We propose an experiment to test our conclusions.

\footnotetext{
${ }^{1}$ Department of Theoretical Physics, National Institute of Physics and Nuclear Engineering, 077125 Bucharest-Măgurele, Romania. ${ }^{2}$ Research Center for Spatial Information - CEOSpaceTech, University Politehnica of Bucharest, 313 Splaiul Independentei, 061071 Bucharest, Romania. ${ }^{3}$ Institute for Quantum Computing, University of Waterloo, Waterloo, Ontario, Canada N2L 3G1. ${ }^{4}$ Department of Physics and Astronomy, University of Waterloo, Waterloo, Ontario, Canada N2L 3G1. ${ }^{5}$ Perimeter Institute for Theoretical Physics, Waterloo, Ontario, Canada N2L 2Y6. ${ }^{6}$ Department of Physics and Astronomy, Macquarie University, Sydney, New South Wales 2109, Australia. Correspondence and requests for materials should be addressed to D.T. (email: daniel.terno@mq.edu.au).
} 
Q uantum mechanics is proverbially counterintuitive ${ }^{1,2}$. For many years, thought experiments were used to dissect its puzzling properties, while hidden-variable (HV) models strived to explain or even to remove them ${ }^{1-4}$. The development of quantum technologies ${ }^{5,6}$ enabled us not only to perform several former gedanken experiments ${ }^{1,2}$, but also to devise new ones ${ }^{7-11}$. One can gain new insights into quantum foundations by introducing quantum controlling devices ${ }^{10-12}$ into well-known experiments. This has led, for example, to a reinterpretation ${ }^{11-14}$ of Bohr's complementarity principle ${ }^{15}$.

Wave-particle duality is best illustrated by the classic Wheeler delayed-choice experiment (WDC) $)^{16-18}$, Fig. 1a,b. A photon enters a Mach-Zehnder interferometer (MZI) and its trajectory is coherently split by the beamsplitter $\mathrm{BS}_{1}$ into an upper and a lower path. The upper path contains a variable phase shift $\varphi$. A random number generator controls the insertion $(b=1)$ or removal $(b=0)$ of a second beamsplitter $\mathrm{BS}_{2}$. If $\mathrm{BS}_{2}$ is present, the interferometer is closed and we observe an interference pattern depending on the phase shift $\varphi$. If $\mathrm{BS}_{2}$ is absent, the MZI is open and the detectors measure a constant probability distribution independent of $\varphi$. Thus, depending on the experimental setup, the photon behaves in two completely different ways. In the case of the closed MZI, the interference pattern suggests that the photon travelled along both paths simultaneously and interfered with itself at the second beamsplitter $\mathrm{BS}_{2}$, hence showing a wavelike behaviour. However, if the interferometer is open, since always only one of the two detectors fires, one is led to the conclusion that the photon travelled only one path, hence displaying a particle-like behaviour.

The complementarity of the interferometer setups required to observe particle or wave behaviour obscures the simultaneous

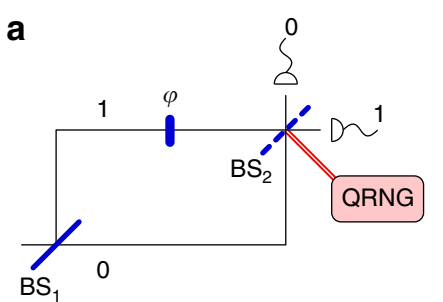

b

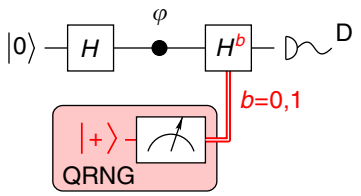

C
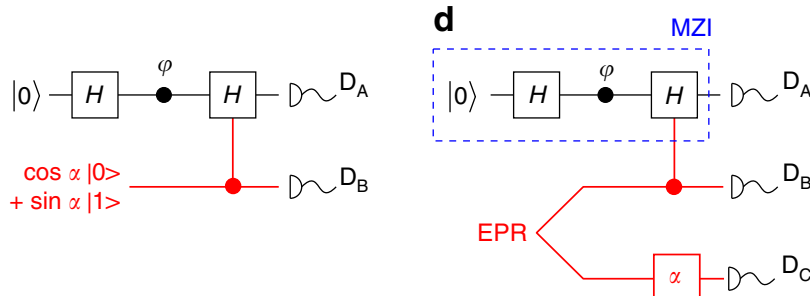

Figure 1 | The evolution of the delayed-choice experiment. (a) In Wheeler's classic experiment, the second beamsplitter is inserted or removed after the photon is inside the interferometer; this prevents the photon from changing its mind ${ }^{16}$ about being a particle or a wave. The detectors observe either an interference pattern depending on the phase $\phi$ (wave behaviour), or an equal distribution of hits (particle behaviour). A quantum random number generator ( $Q R N G$ ) determines whether $\mathrm{BS}_{2}$ is inserted or not. Quantum networks: (b) in the classic delayed-choice experiment the QRNG is an auxiliary quantum system initially prepared in the equal superposition state $|+\rangle=\frac{1}{\sqrt{2}}(|0\rangle+|1\rangle)$ and then measured. The Hadamard gate $H$ is the quantum network equivalent of the beamsplitter; (c) delayed choice with a quantum control ${ }^{11}$; (d) entanglement-

assisted quantum delayed-choice experiment ${ }^{19}$. The ancilla $C$ is measured along the direction $-\alpha$, equivalent to the application of a rotation $R_{y}(\alpha)=$ $e^{i \alpha \sigma_{y}}$ before a measurement in the computational basis. presence of both properties, allowing the (objective) view that, at any moment of time, a photon can be either a particle or a wave. The WDC experiment uncovers the difficulty inherent in this view by randomly choosing whether or not to insert the second beamsplitter $\left(\mathrm{BS}_{2}\right)$ after the photon enters the interferometer (Fig. 1a). This delayed choice prevents a possible causal link between the experimental setup and the photon's behaviour: the photon should not know beforehand if it has to behave like a particle or like a wave.

The delayed-choice experiment with a quantum control (Fig. 1c) highlights the complexity of space-time ordering of events, once parts of the experimental setup become quantum systems ${ }^{11}$. The quantum-controlled delayed-choice experiment has been recently implemented in several different systems ${ }^{19-23}$. To ensure the quantum behaviour of the controlling device, one can either test the Bell inequality ${ }^{23}$ or use an entangled ancilla ${ }^{19}$.

The theoretical analysis of the quantum WDC involved so far a single binary HV $\lambda$ describing the classical concepts of wave/ particle. Here, we introduce a full HV description for both the photon A and the ancilla. We analyse the relationships between the concepts of determinism, wave-particle objectivity and local independence of $\mathrm{HV}$ in the entanglement-controlled delayedchoice experiment. We show that, when combined, these assumptions lead to predictions that are different from those of quantum mechanics, even if any two of them are compatible with it. We propose and discuss an experiment to test our conclusions.

\section{Results}

Notation. We use the conventions as in refs 3,$12 ; q(a, b, \ldots)$ are the quantum-mechanical probability distributions and $p(a, b, \ldots, \Lambda)$ the predictions of $\mathrm{HV}$ theories with a $\mathrm{HV} \Lambda$. We consider either a single HV $\Lambda$, which fully determines behaviour of the system, or refine it as $\Lambda_{1}, \Lambda_{2}$ pertaining to different parts of the system. For simplicity, we assume $\Lambda$ is discrete; the analysis can be easily generalized to the continuous case.

Quantum system. The system we analyse consists of three qubits: a photon $\mathrm{A}$ and an entangled pair BC (Fig. 1d). We denote the measurement outcomes for the photon $\mathrm{A}$ as $a=0,1$ and for the two ancilla qubits as $b$ and $c$; the corresponding detectors are $\mathrm{D}_{\mathrm{A}}$, $\mathrm{D}_{\mathrm{B}}$ and $\mathrm{D}_{\mathrm{C}}$. The system is prepared in the initial state $|0\rangle_{\mathrm{A}}(\sqrt{\eta}|00\rangle+\sqrt{1-\eta}|11\rangle)_{\mathrm{BC}}$; for $\eta=\frac{1}{2}$, $\mathrm{BC}$ is a maximally entangled EPR pair.

Photon A enters a MZI in which the second beamsplitter is quantum-controlled by qubit B. The third qubit $\mathrm{C}$ undergoes a $\sigma_{y}$ rotation $R_{y}(\alpha)=e^{i \alpha \sigma_{y}}$ followed by a measurement in the computational basis. The state before the measurements is

$$
\begin{aligned}
|\psi\rangle= & (\sqrt{\eta} \cos \alpha|\mathrm{p}\rangle|0\rangle+\sqrt{1-\eta} \sin \alpha|\mathrm{w}\rangle|1\rangle)_{\mathrm{AB}}|0\rangle_{\mathrm{C}} \\
& -(\sqrt{\eta} \sin \alpha|\mathrm{p}\rangle|0\rangle-\sqrt{1-\eta} \cos \alpha|\mathrm{w}\rangle|1\rangle)_{\mathrm{AB}}|1\rangle_{\mathrm{C}} .
\end{aligned}
$$

The counting statistics that result from the particle-like state $|\mathrm{p}\rangle=\frac{1}{\sqrt{2}}\left(|0\rangle+e^{i \varphi}|1\rangle\right)$ and the wave-like state $|\mathrm{w}\rangle=$ $e^{i \varphi / 2}\left(\cos \frac{\varphi}{2}|0\rangle-i \sin \frac{\varphi}{2}|1\rangle\right)$ are discussed below (equations (3 and 4) and Methods).

Constraints on HV theories. Our strategy is to show that $q(a, b$, c) cannot result from a probability distribution $p(a, b, c, \Lambda)$ of a $\mathrm{HV}$ theory satisfying the requirements of wave-particle objectivity, local independence and determinism. Any viable HV theory should satisfy the adequacy condition: namely, it should reproduce the quantum statistics by summing over all HVs $\Lambda$ :

$$
q(a, b, c)=p(a, b, c)=\sum_{\Lambda} p(a, b, c, \Lambda) .
$$




\section{Box 1 | Three classical assumptions.}

Wave-particle objectivity. We define particles and waves according to the experimental behaviour in an open, respectively closed, MZI ${ }^{11}$. A particle in an open interferometer $(b=0)$ is insensitive to the phase shift in one of the arms and therefore has the statistics

$$
p(a \mid b=0, \Lambda)=\left(\frac{1}{2}, \frac{1}{2}\right), \quad \forall \Lambda \in \mathcal{L}_{\mathrm{p}} .
$$

In contrast, a wave in a closed MZI $(b=1)$ shows interference

$$
p(a \mid b=1, \Lambda)=\left(\cos ^{2} \frac{\varphi}{2}, \sin ^{2} \frac{\varphi}{2}\right), \quad \forall \Lambda \in \mathcal{L}_{\mathrm{w}} .
$$

The sets $\mathcal{L}_{\mathrm{p}}$ and $\mathcal{L}_{\mathrm{w}}$ must be disjoint; otherwise, there are values of $\Lambda$ that introduce wave-particle duality. Writing $\mathcal{L}_{\mathrm{p}} \cup \mathcal{L}_{\mathrm{w}}=\mathcal{L}_{\text {, }}$ the wave/ particle property is expressed by a mapping $\lambda: \mathcal{L} \mapsto\{p, w\}$ and the sets $\mathcal{L}_{\mathrm{p}}=\lambda^{-1}(p), \mathcal{L}_{\mathrm{w}}=\lambda^{-1}(\mathrm{w})$ are the pre-images of $p, w$ under the function $\lambda$.

Determinism. The HV $\Lambda$ determines the individual outcomes of the detection ${ }^{3}$. Specifically, for the setup of (Fig. 1d)

$$
p(a, b, c \mid \Lambda)=\chi_{a b c}(\Lambda)
$$

where the indicator function $\chi=1$, if $\Lambda$ belongs to some predetermined set, and $\chi=0$ otherwise.

Local independence. The HV $\Lambda$ are split into $\Lambda_{1}$ and $\Lambda_{2}$, and the prior probability distribution has a product structure

$$
p(\Lambda)=f\left(\Lambda_{1}\right) F\left(\Lambda_{2}\right),
$$

for some probability distributions $f$ and $F$, where the subscripts 1 and 2 , respectively, refer to the photon $A$ and the pair BC. Such bilocal variables have been previously considered in ref. 29.

We encapsulate the additional classical expectations into three assumptions (see Box 1 for the formal definitions of the concepts we consider in this section).

For a given photon, we require the property of being a particle or a wave to be objective (intrinsic), that is, to be unchanged during its lifetime. This condition selects from the set of adequate $\mathrm{HV}$ theories those models that have meaningful notions of particle and wave ${ }^{11}$. For each photon, the $\mathrm{HV} \Lambda$ should determine unambiguously if the photon is a particle or a wave, thus allowing the partition of the set of HVs $\mathcal{L}$ into two disjoint subsets, $\mathcal{L}=\mathcal{L}_{\mathrm{p}} \cup \mathcal{L}_{\mathrm{w}}$, where the subscript indicates the property, particle or wave.

The particle (wave) properties are abstractions of the particle (wave) counting statistics in open (closed) MZI, respectively. The behaviour of a particle (wave) in a closed (open) MZI is not constrained; this allows for significant freedom in constructing HV theories. Experimentally, the wave or particle behaviour depends only on the photon and the settings of the MZI:

$$
p(a \mid b, c, \Lambda)=p(a \mid b, \Lambda)
$$

for all values of $a, b, c$ and $\Lambda$.

By replacing the single-qubit ancilla with an entangled pair, one can take advantage of both the quantum control and the space-like separation between events. The rationale behind the third qubit $\mathrm{C}$ is that it allows us to choose the rotation angle $\alpha$ after both qubits $\mathrm{A}$ (the photon) and B (the quantum control) are detected. This is not possible in the standard quantum $\mathrm{WDC}^{11}$, Fig. 1c, where the quantum control B has to be prepared (by setting the angle $\alpha$ ) before it interacts with A. As discussed in Methods, there is a unique assignment of probabilities that satisfies all the requirements of adequacy, wave-particle objectivity and determinism. Adopting this assignment, we reach the same level of incongruity as in ref. 11, since the probability $p(\lambda)$ of photon A being a particle or a wave is determined by the entanglement between $\mathrm{B}$ and $\mathrm{C}$,

$$
p(\lambda)=(\eta, 1-\eta)
$$

This incongruity becomes an impossibility when the photon A and the entangled pair BC are prepared independently. In this case, their HVs are generated independently; that is, a single $\mathrm{HV}$ $\Lambda$ not only has the structure $\Lambda=\left(\Lambda_{1}, \Lambda_{2}\right)$, where the subscripts 1 and 2 refer to the photon $\mathrm{A}$ and the pair $\mathrm{BC}$, respectively, but the prior probability distribution of $\mathrm{HV}$ has a product form. To realize this condition experimentally, we rely on the absence of the superluminal communication and a space-like separation of the two events.

Unlike the typical Bell-inequality scenarios, we have a single measurement setup which involves two independent $\mathrm{HV}$ distributions. Moreover, by performing the rotation $R_{y}(\alpha)$ and the detection $\mathrm{D}_{\mathrm{C}}$ sufficiently fast, such that the information about A and $\Lambda_{1}$ cannot reach the detector $\mathrm{D}_{\mathrm{C}}$, the detection outcome is determined only by $\Lambda_{2}$. Since being a wave (particle) is assumed to be an objective property of $\mathrm{A}, \lambda=\lambda\left(\Lambda_{1}\right)$ is a binary function of the $\mathrm{HV} \Lambda_{1}$ only.

Contradiction. We show in Methods that for $\eta \neq 0,1$ (these two cases correspond to an always closed or opened MZI), the requirements of adequacy, wave-particle objectivity, determinism and local independence are satisfied only if

$$
\cos 2 \alpha=0
$$

This proves our main theoretical result: determinism, local independence and wave-particle objectivity are not compatible with quantum mechanics for any $\alpha \neq \pm \pi / 4, \pm 3 \pi / 4$. We will later discuss how exactly a HV theory that satisfies the three classical assumptions is inadequate.

Proposed experiment. In Fig. 2, we show the proposed experimental setup for the entanglement-controlled delayed-choice experiment. Two pump pulses (blue) are incident on two nonlinear crystals and generate via spontaneous parametric downconversion two pairs of entangled photons (red). One of the photons is the trigger and the other three are the photons $\mathrm{A}, \mathrm{B}, \mathrm{C}$, with $\mathrm{BC}$ being the entangled pair.

Photons $\mathrm{A}$ and $\mathrm{B}$ are held in the lab (with appropriate delay lines) and together they implement the controlled MZI. The central element is the quantum switch, which is the controlledHadamard gate $C(H)=(W \otimes I) C(Z)(W \otimes I)$, where $W=\sigma_{z} e^{i \frac{\pi}{8} \sigma_{y}}$. The photonic controlled- $Z$ gate $C(Z)$ is implemented with a partially polarizing beamsplitter and is done probabilistically via post-selection ${ }^{24,25}$. Optical wave plates perform single-qubit rotations (gates $H, \varphi$ and $W$ ) on photon A. Photon $\mathrm{C}$ is sent through a channel at a distant location, then measured in a rotated basis. Two independent lasers generate the two photon pairs (Fig. 2 (refs 26,27)); in this case, we can use equation (6) to describe independent probability distributions for $\Lambda_{1}$ and $\Lambda_{2}$.

\section{Discussion}

In this section, we consider how exactly a HV theory, which satisfies the three classical assumptions, fails the adequacy test. The interference pattern measured by the detector $\mathrm{D}_{\mathrm{A} 0}$ is $I_{\mathrm{A}}(\varphi)=\operatorname{Tr}\left(\rho_{\mathrm{A}}|0\rangle\langle 0|\right)$, with $\rho_{\mathrm{A}}=\operatorname{Tr}_{\mathrm{BC}}|\psi\rangle\langle\psi|$, the reduced density matrix of photon $A$. The data can be postselected according to the outcome $c$ resulting in $I_{\mathrm{A} \mid c}$. The visibility of the interference pattern (Methods) is $V=\left(I_{\max }-I_{\min }\right) /\left(I_{\max }+I_{\min }\right)$, where the $\mathrm{min} / \mathrm{max}$ values are calculated with respect to $\varphi$. 


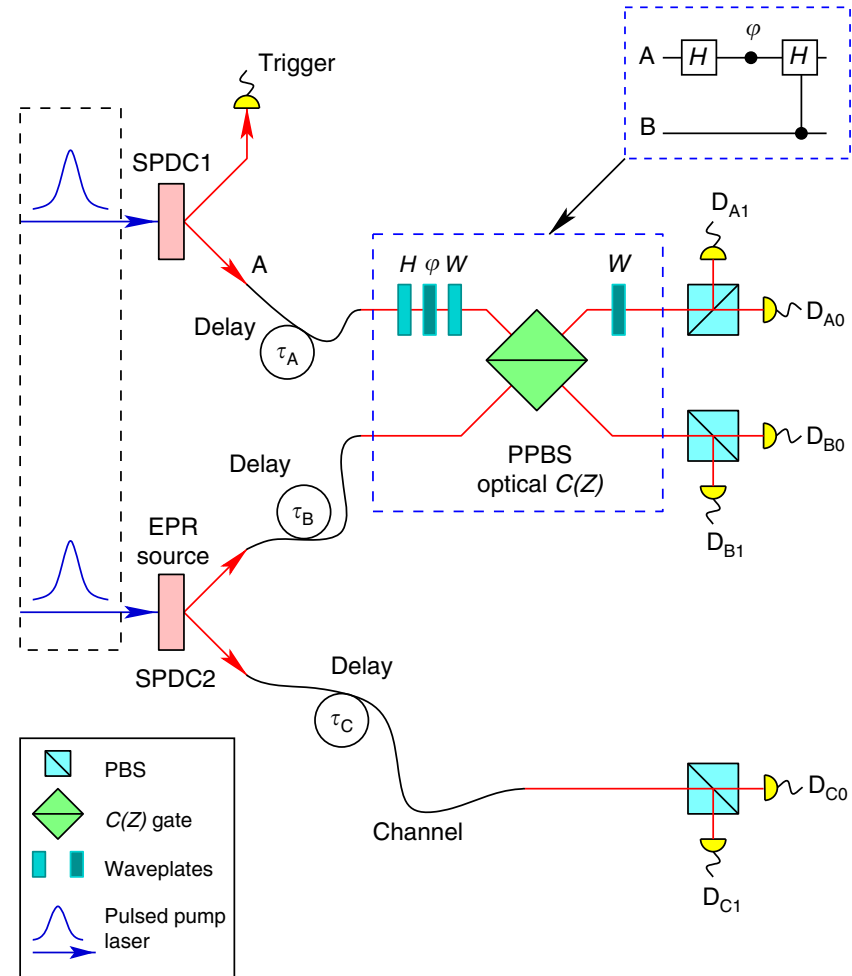

Figure 2 | Proposed experimental setup. Two space-like separated pump pulses (blue) generate, via spontaneous parametric down-conversion, two pairs of entangled photons (red). The first photon is the trigger and the other three the photons A, B, C. Inset: the quantum-controlled MZI. The optical delays in the three photon arms, $\tau_{A}, \tau_{B}, \tau_{C}$ can be adjusted to ensure the desired time ordering of the detection events.

The postselected visibility for $c=0$ is (Fig. 3)

$$
V_{\mathrm{A} \mid c=0}=\frac{(1-\eta) \sin ^{2} \alpha}{\eta \cos ^{2} \alpha+(1-\eta) \sin ^{2} \alpha}
$$

The full (non-postselected) visibility is $V_{\mathrm{A}}=1-\eta$ and gives information about the initial entanglement of the $\mathrm{BC}$ pair. On the other hand, if one assumes that the $\mathrm{HV}$ are distributed according to equation (6) and satisfy the wave-particle objectivity and determinism, the visibility is independent of $c$,

$$
V_{\mathrm{A}}^{\mathrm{HV}} \equiv V_{\mathrm{A} \mid c=0}^{\mathrm{HV}}=V_{\mathrm{A} \mid c=1}^{\mathrm{HV}}=1-f,
$$

in contrast with the quantum-mechanical prediction (Fig. 3). Details of this calculation are in Methods.

This incompatibility between the basic tenets of HV theories and quantum mechanics has two remarkable features. First, the contradiction is revealed for any, arbitrarily small, amount of entanglement. This test is in sharp distinction with Bell-type experiments insofar as our result is free from inequalities. Waveparticle objectivity, revealed only statistically, is more intuitive and technically milder than the assumption of sharp values of quantum incompatible observables. Second, in our setup, any two of the classical ideas together are compatible with the quantummechanical predictions. This fact, and the way we arrived at the contradiction, invite questions concerning the internal consistency of classical concepts ${ }^{28}$.

\section{Methods}

Quantum-mechanical analysis. The initial state of photons A, B and C is

$$
|0\rangle_{\mathrm{A}}(\sqrt{\eta}|00\rangle+\sqrt{1-\eta}|11\rangle)_{\mathrm{BC}} .
$$

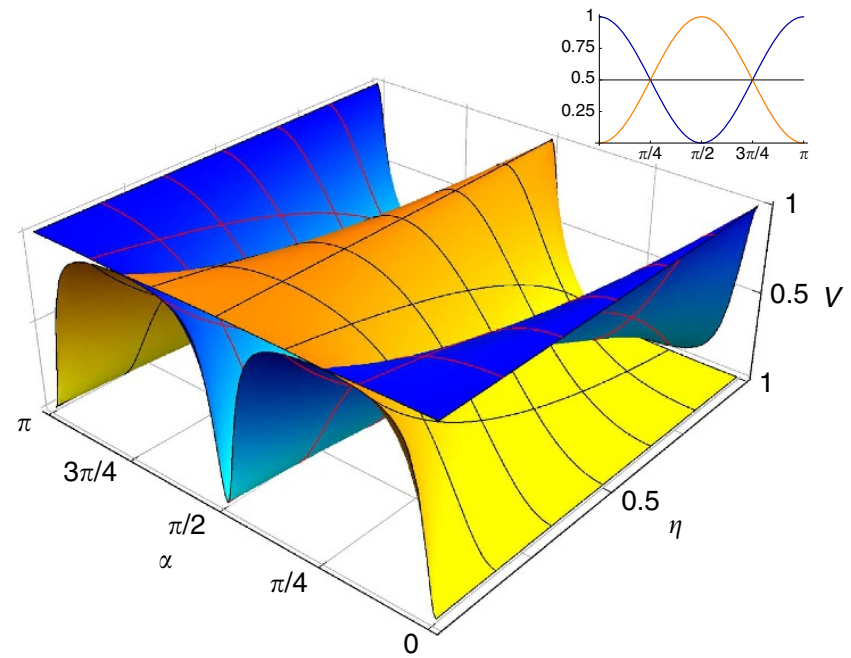

Figure 3 | Visibility. The visibilities $V_{\mathrm{A} \mid c=0}$ (yellow) and $V_{\mathrm{A} \mid \mathrm{c}=1}$ (blue), are calculated in Methods. In HV theories, the visibility does not distinguish between the $c=0,1$ cases. The inset illustrates this for $p(p)=\eta=\frac{1}{2^{\prime}}$ with the straight line representing the HV visibility prediction.

The ancilla qubits B and C are maximally entangled for $\eta=\frac{1}{2}$. The final state before measurement is given by equation (1). From it, we calculate the quantum statistics $q(a, b, c)$, where each of $a, b$, and $c$ take the values $\{0,1\}$. The probability distribution for $c=0$ is

$$
\begin{aligned}
q(a, b, c=0)= & \left(\frac{1}{2} \eta \cos ^{2} \alpha,(1-\eta) \sin ^{2} \alpha \cos ^{2} \frac{\varphi}{2},\right. \\
& \left.\frac{1}{2} \eta \cos ^{2} \alpha,(1-\eta) \sin ^{2} \alpha \sin ^{2} \frac{\varphi}{2}\right) .
\end{aligned}
$$

where the four entries correspond to the values $(a, b)=(00,01,10,11)$. For $c=1$, we obtain

$$
\begin{aligned}
q(a, b, c=1)= & \left(\frac{1}{2} \eta \sin ^{2} \alpha,(1-\eta) \cos ^{2} \alpha \cos ^{2} \frac{\varphi}{2},\right. \\
& \left.\frac{1}{2} \eta \sin ^{2} \alpha,(1-\eta) \cos ^{2} \alpha \sin ^{2} \frac{\varphi}{2}\right) .
\end{aligned}
$$

This in turn yields

$$
\begin{gathered}
q(a, b)=\left(\frac{1}{2} \eta,(1-\eta) \cos ^{2} \frac{\varphi}{2}, \frac{1}{2} \eta,(1-\eta) \sin ^{2} \frac{\varphi}{2}\right), \\
q(b, c)=\left(\eta \cos ^{2} \alpha, \eta \sin ^{2} \alpha,(1-\eta) \sin ^{2} \alpha,(1-\eta) \cos ^{2} \alpha\right), \\
q(b)=(\eta, 1-\eta), \\
q(c)=\left(\eta \cos ^{2} \alpha+(1-\eta) \sin ^{2} \alpha, \eta \sin ^{2} \alpha+(1-\eta) \cos ^{2} \alpha\right) .
\end{gathered}
$$

For $\eta=\frac{1}{2}$, the probability distributions for $b$ and $c$ are equal. If $\eta \neq \frac{1}{2}$, B and C are no longer maximally entangled and the symmetry between them is broken: a rotation $\alpha$ on $\mathrm{C}$ no longer corresponds to a rotation $\alpha$ on $\mathrm{B}$. The conditional probabilities are

$$
q(c \mid b)=\left(\cos ^{2} \alpha, \sin ^{2} \alpha, \sin ^{2} \alpha, \cos ^{2} \alpha\right),
$$

and from Bayes' rule $q(b \mid c)=q(c \mid b) q(b) / q(c)$.

Solution to the three constraints. We now show that it is possible to construct a $\mathrm{HV}$ model that is adequate, objective and deterministic. The unknown parameters at our disposal are 16 probabilities $p(a, b, c, \lambda)$. These probabilities are derived from the underlying distribution $p(\Lambda)$ summed over appropriate domains. At this stage, we do not enquire about the connection with the HV $\Lambda$. The probabilities $p(a, b, c$, i) satisfy seven adequacy constraints, equations (13) and (14), plus the normalization constraint. The adequacy conditions can be written as

$$
q(a, b, c)=p(a, b, c)=p(a, b, c, \mathrm{p})+p(a, b, c, \mathrm{w}) .
$$

In addition, equation (7) and the standard rules for the conditional probabilities, such as

$$
p(a \mid b, \lambda) \equiv p(a \mid b, c, \lambda)=\frac{p(a, b, c, \lambda)}{p(0, b, c, \lambda)+p(1, b, c, \lambda)},
$$

imply the existence of four additional constrains,

$$
p(0,0, c, \mathrm{p})=p(1,0, c, \mathrm{p}),
$$

$$
p(0,1, c, \mathrm{w}) \sin ^{2} \frac{\varphi}{2}=p(1,1, c, \mathrm{w}) \cos ^{2} \frac{\varphi}{2} .
$$


The resulting linear system has a four-parameter family of solutions. However, a straightforward calculation shows that for all these solutions $p_{4}(a, b, c, \lambda)$, the resulting statistics in an open/closed MZI is independent of $\lambda$,

$$
\begin{gathered}
p_{4}(a \mid b=0, \mathrm{p})=p_{4}(a \mid b=0, \mathrm{w})=\left(\frac{1}{2}, \frac{1}{2}\right), \\
p_{4}(a \mid b=1, \mathrm{w})=p_{4}(a \mid b=1, \mathrm{p})=\left(\cos ^{2} \frac{\varphi}{2}, \sin ^{2} \frac{\varphi}{2}\right),
\end{gathered}
$$

that is, the statistics of $\mathrm{D}_{\mathrm{A}}$ is determined solely by the state of the interferometer.

We can avoid the reintroduction of wave-particle duality using a special solution

$$
p_{\mathrm{s}}(b \mid \lambda)=\delta_{\lambda_{\mathrm{p}}} \delta_{b 0}+\delta_{\lambda_{\mathrm{w}}} \delta_{b 1} \equiv p_{\mathrm{s}}(\lambda \mid b),
$$

which imposes the $b-\lambda$ correlation (compare ref. 11). As a result,

$$
p(b=0, \lambda=\mathrm{w})=p(b=1, \lambda=\mathrm{p})=0,
$$

and since the probabilities are positive,

$$
\sum_{a, c} p(a, 1, c, \mathrm{p})=\sum_{a, c} p(a, 0, c, \mathrm{w})=0,
$$

the eight above probabilities are zero individually. The system appears overconstrained, but it still has a unique solution

$$
p_{\mathrm{s}}(a, b, c, \lambda)=q(a, b, c) p_{\mathrm{s}}(b \mid \lambda) .
$$

In particular,

$$
p_{\mathrm{s}}(\lambda)=\sum_{a, b, c} p_{\mathrm{s}}(a, b, c, \lambda)=(\eta, 1-\eta) .
$$

Deriving the contradiction. In addition to the partition of $\mathcal{L}$ according to the values of $\lambda=\mathrm{p}$, w, we will use the decomposition of the set of $\mathrm{HV}$ according to the outcomes of $\mathrm{D}_{\mathrm{C}}$. The two branches $c=0,1$ correspond to the partition

$$
\mathcal{L}=\mathcal{L}_{0} \cup \mathcal{L}_{1},
$$

where for $\Lambda \in \mathcal{L}_{c}$, the outcome of $\mathrm{D}_{\mathrm{C}}$ is $c$. The assumption of local independence implies a Cartesian product structure

$$
\mathcal{L}=\left\{\Lambda_{1}\right\} \times\left(\mathcal{L}_{0}^{2} \cup \mathcal{L}_{1}^{2}\right)=\left(\mathcal{L}_{\mathrm{p}}^{1} \cup \mathcal{L}_{\mathrm{w}}^{1}\right) \times\left(\mathcal{L}_{0}^{2} \cup \mathcal{L}_{1}^{2}\right),
$$

of the set of HV, where the subsets depend on the experimental setup. When the superscripts 1 and 2 on $\mathcal{L}$ are redundant, we may omit them.

Now, we show that under the assumptions of adequacy and the three classical assumptions of the wave-particle objectivity, determinism and local independence, it is impossible to derive the solution $p(a, b, c, \lambda)$ with any arrangement of the probabilities $p(\Lambda)$. The probability of the outcome $c$ satisfies

$$
q(c) \equiv p(c)=\sum_{\Lambda \in \mathcal{L}_{c}} p(\Lambda)=\sum_{\Lambda \in \mathcal{\mathcal { L } _ { \mathrm { p } }} \cap \mathcal{L}_{c}} p(\Lambda)+\sum_{\Lambda \in \mathcal{L}_{\mathrm{w}} \cap \mathcal{L}_{c}} p(\Lambda) .
$$

To simplify the calculations, we enumerate the variables $\Lambda_{1,2}$ by the indices $i, j$, respectively. The domain $\mathcal{L}_{c}^{2}$ corresponds, according to the hypothesis, to the index set $J_{c}$ of $\Lambda_{2}$, and the domains $\mathcal{L}_{\mathrm{p}}^{1}$ and $\mathcal{L}_{\mathrm{w}}^{1}$ to the index sets $I_{\mathrm{p}}$ and $I_{\mathrm{w}}$ of $\Lambda_{1}$, respectively. In particular,

$$
p(\lambda)=\left(\sum_{i \in I_{\mathrm{p}}} f_{i}, \sum_{i \in I_{\mathrm{w}}} f_{i}\right),
$$

for some $f_{i} \equiv f\left(\Lambda_{1}^{i}\right)$. The prior distribution of HV and the domains of summation can depend on the parameters $\eta, \varphi$ and $\alpha$.

The putative behaviour of a wave $(\lambda=\mathrm{w})$ in an open $(b=0)$ interferometer and of a particle $(\lambda=\mathrm{p})$ in a closed $(b=1)$ one is characterized by two unknown distributions $x_{i j}, i \in I_{\mathrm{w}}$ and $y_{i j}, i \in I_{\mathrm{p}}$, respectively

$$
\begin{array}{ll}
p\left(a \mid b=0, \Lambda=\left(\Lambda_{1}^{i}, \Lambda_{2}^{j}\right)\right)=\left(x_{i j}, 1-x_{i j}\right), & i \in I_{\mathrm{p}} \\
p\left(a \mid b=1, \Lambda=\left(\Lambda_{1}^{i}, \Lambda_{2}^{j}\right)\right)=\left(y_{i j}, 1-y_{i j}\right), & i \in I_{\mathrm{w}}
\end{array}
$$

allowing for a possible dependence on a value of $\Lambda_{2}$. The remaining two sets of variables are the probability distributions for $b$ conditioned on the values of HVs $\Lambda$ :

$$
\begin{array}{ll}
p\left(b \mid \Lambda=\left(\Lambda_{1}^{i}, \Lambda_{2}^{j}\right)\right)=\left(z_{i j}, 1-z_{i j}\right), & i \in I_{\mathrm{p}}, \\
p\left(b \mid \Lambda=\left(\Lambda_{1}^{i}, \Lambda_{2}^{j}\right)\right)=\left(v_{i j}, 1-v_{i j}\right), & i \in I_{\mathrm{w}} .
\end{array}
$$

The requirement of adequacy means that the proposed HV theory reproduces the quantum statistics given above. For compactness, we refer to the probability of having the HV values $\left(\Lambda_{1}=\Lambda_{1}^{i}, \Lambda_{2}=\Lambda_{2}^{j}\right), p\left(\Lambda_{1}^{i}, \Lambda_{2}^{j}\right)$, as $p_{i j}$, using the same convention as for $x_{i j}, y_{i j}, z_{i j}$ and $v_{i j}$. For $c=0$, we have

$$
q(0,0,0)=\frac{1}{2} \eta \cos ^{2} \alpha \equiv \frac{1}{2} \sum_{i \in I_{\mathrm{p}}, j \in J_{0}} z_{i j} p_{i j}+\sum_{i \in I_{\mathrm{w}}, j \in J_{0}} x_{i j} p_{i j} p_{i j},
$$

$$
\begin{aligned}
q(0,1,0) & =(1-\eta) \sin ^{2} \alpha \cos ^{2} \frac{\varphi}{2} \\
& \equiv \sum_{i \in I_{\mathrm{p}}, j \in J_{0}} y_{i j}\left(1-z_{i j}\right) p_{i j}+\cos ^{2} \frac{\varphi}{2} \sum_{i \in I_{\mathrm{w}}, j \in J_{0}}\left(1-v_{i j}\right) p_{i j},
\end{aligned}
$$

$$
q(1,0,0)=\frac{1}{2} \eta \cos ^{2} \alpha \equiv \frac{1}{2} \sum_{i \in I_{\mathrm{p}}, j \in J_{0}} z_{i j} p_{i j}+\sum_{i \in I_{\mathrm{w}}, j \in J_{0}}\left(1-x_{i j}\right) v_{i j} p_{i j}
$$

$$
\begin{aligned}
q(1,1,0) & =(1-\eta) \sin ^{2} \alpha \sin ^{2} \frac{\varphi}{2} \\
& \equiv \sum_{i \in I_{\mathrm{p}}, j \in J_{0}}\left(1-y_{i j}\right)\left(1-z_{i j}\right) p_{i j}+\sin ^{2} \frac{\varphi}{2} \sum_{i \in I_{\mathrm{w}}, j \in J_{0}}\left(1-v_{i j}\right) p_{i j},
\end{aligned}
$$

with analogous expressions for $c=1$. Adding and subtracting equations (39) and (41) we obtain, respectively

$$
\begin{gathered}
\sum_{i \in I_{\mathrm{p}}, j \in J_{0}} z_{i j} p_{i j}+\sum_{i \in I_{w}, j \in J_{0}} v_{i j} p_{i j}=\eta \cos ^{2} \alpha \\
\sum_{i \in I_{\mathrm{w}}, j \in J_{0}}\left(1-2 x_{i j}\right) v_{i j} p_{i j}=0
\end{gathered}
$$

Adding equations (40) and (42) yields

$$
(1-\eta) \sin ^{2} \alpha=\sum_{i \in I_{\mathrm{p}}, j \in J_{0}}\left(1-z_{i j}\right) p_{i j}+\sum_{i \in I_{\mathrm{w}}, j \in J_{0}}\left(1-v_{i j}\right) p_{i j},
$$

which on substitution back into equation (40) results in

$$
\sum_{i \in I_{\mathrm{p}}, j \in J_{0}}\left(\cos ^{2} \frac{\varphi}{2}-y_{i j}\right)\left(1-z_{i j}\right) p_{i j}=0 .
$$

Four additional equations (giving a total of seven independent equations) are obtained for $j \in J_{1}$ with $\cos ^{2} \alpha \leftrightarrow \sin ^{2} \alpha$

From equation (26), it follows that $v_{i j}=0, i \in I_{\mathrm{w}}$ and $z_{i j}=1, i \in I_{\mathrm{p}}$. Hence, for $c=0$, only two equations are not automatically satisfied,

$$
\eta \cos ^{2} \alpha=\sum_{i \in I_{\mathrm{p}}, j \in J_{0}} p_{i j}, \quad(1-\eta) \sin ^{2} \alpha=\sum_{i \in I_{w}, j \in J_{0}} p_{i j} .
$$

The corresponding equations for $c=1$ are

$$
\eta \sin ^{2} \alpha=\sum_{i \in I_{\mathrm{p}}, j \in I_{1}} p_{i j}, \quad(1-\eta) \cos ^{2} \alpha=\sum_{i \in I_{w}, j \in I_{1}} p_{i j},
$$

which are in agreement with $q(c)$, equation (18).

Now we use the product structure of the probability distribution, equation (6),

$$
p(\Lambda)=f\left(\Lambda_{1}\right) F\left(\Lambda_{2}\right)_{i j}=f_{i} F_{j} .
$$

Using equations (28) and (30), we find that

$$
\sum_{i \in I_{\mathrm{p}}} f_{i}=\eta
$$

Adding the pairs of equations in (47) and (48) and summing over the index $i$, we express the adequacy condition $q(c)=\sum_{j \in I_{c}} F_{j}$,

$$
\begin{aligned}
& \eta \cos ^{2} \alpha+(1-\eta) \sin ^{2} \alpha=\sum_{j \in J_{0}} F_{j}, \\
& \eta \sin ^{2} \alpha+(1-\eta) \cos ^{2} \alpha=\sum_{j \in J_{1}} F_{j},
\end{aligned}
$$

but on the other hand, for $\eta \neq 0,1$ summing over $i$ in each of these four equations separately and using equation (50) we get

$$
\sum_{j \in J_{0}} F_{j}=\cos ^{2} \alpha=\sin ^{2} \alpha=\sum_{j \in I_{1}} F_{j} .
$$

These equations can be satisfied for any $\eta$ only if

$$
\cos ^{2} \alpha=\sin ^{2} \alpha,
$$

resulting in the contradiction (for arbitrary $\alpha$ ) $\cos 2 \alpha=0$.

Experimental signature. The interference pattern measured by the detector $\mathrm{D}_{\mathrm{A}}$ is $I_{\mathrm{A}}(\varphi)=\operatorname{Tr}\left(\rho_{A}|0\rangle\langle 0|\right)$, with $\rho_{A}=\operatorname{Tr}_{B C}|\psi\rangle\langle\psi|$ the reduced density matrix of photon A. The data can be postselected according to the outcome $c$ resulting in $I_{\mathrm{A} \mid c \text {. }}$ The intensity (signal) measured by detector $\mathrm{D}_{\mathrm{A}}$ for $c=0$ (and no post-selection on $b$ ) is:

$$
I_{\mathrm{A} \mid c=0}=\frac{1}{2} \eta \cos ^{2} \alpha+(1-\eta) \sin ^{2} \alpha \cos ^{2} \frac{\varphi}{2},
$$


giving the visibility

$$
V_{\mathrm{A} \mid c=0}=\frac{(1-\eta) \sin ^{2} \alpha}{\eta \cos ^{2} \alpha+(1-\eta) \sin ^{2} \alpha} .
$$

A similar calculation gives the visibility for $c=1$

$$
V_{\mathrm{A} \mid c=1}=\frac{(1-\eta) \cos ^{2} \alpha}{\eta \sin ^{2} \alpha+(1-\eta) \cos ^{2} \alpha} .
$$

The full intensity measured by detector $\mathrm{D}_{\mathrm{A}}$ (without postselecting on $c$ ) is $I_{\mathrm{A}}=\frac{1}{2} \eta+(1-\eta) \cos ^{2} \frac{\varphi}{2}$ and the corresponding visibility

$$
V_{\mathrm{A}}=1-\eta \text {. }
$$

Thus the visibility of detector $\mathrm{D}_{\mathrm{A}}$ gives information about the entanglement of the $\mathrm{BC}$ pair.

We now calculate the visibilities predicted by a non-trivial HV theory that is assumed to satisfy the three classical assumptions. Using equation (26), we rewrite the counting statistics as

$$
\begin{gathered}
p(0,0,0)=\frac{1}{2} \sum_{i \in I_{\mathrm{p}}, j \in J_{0}} f_{i} F_{j}, \\
p(0,0,1)=\frac{1}{2} \sum_{i \in I_{\mathrm{p}}, j \in J_{1}} f_{i} F_{j} \\
p(0,1,0)=\cos ^{2} \frac{\varphi}{2} \sum_{i \in I_{\mathrm{w}}, j \in J_{0}} f_{i} F_{j}, \\
p(0,1,1)=\cos ^{2} \frac{\varphi}{2} \sum_{i \in I_{\mathrm{w}}, j \in J_{0}} f_{i} F_{j} .
\end{gathered}
$$

For the product probability distribution above, we get

$$
\begin{gathered}
p(0,0 \mid j)=\frac{p(0,0, j)}{p(c=j)}=\frac{\frac{1}{2} \sum_{i \in I_{\mathrm{p}}, k \in J_{j}} f_{i} F_{k}}{\sum_{k \in J_{j}} F_{k}}=\frac{1}{2} f \\
p(0,1 \mid j)=\frac{p(0,1, j)}{p(c=j)}=\frac{\cos ^{2} \frac{\varphi}{2}}{\sum_{i \in I_{\mathrm{w}}, k \in J_{j}} f_{i} F_{k}}=\cos ^{2} \frac{\varphi}{2}(1-f)
\end{gathered}
$$

for $j=0,1$ separately, where $f=\sum_{i \in I_{\mathrm{p}}} f_{i}$. As a result,

$$
I_{\mathrm{A} \mid c=0}^{\mathrm{HV}}=I_{\mathrm{A} \mid c=1}^{\mathrm{HV}}=\frac{1}{2} f+\cos ^{2} \frac{\varphi}{2}(1-f)
$$

giving

$$
V_{\mathrm{A} \mid c=0}^{\mathrm{HV}}=V_{\mathrm{A} \mid c=1}^{\mathrm{HV}}=1-f,
$$

for the visibilities in HV theories.

\section{References}

1. Wheeler, J. A. \& Zurek, W. H. (eds) Quantum Theory and Measurement (Princeton Univ. Press, 1984).

2. Peres, A. Quantum Theory: Concepts and Methods (Kluwer, 1995).

3. Brandenburger, A. \& Yanofsky, N. A. Classification of hidden-variable properties. J. Phys. A 41, 425302 (2008).

4. Englert, B.-G. On quantum theory. Eur. J. Phys. D 67, 238 (2013).

5. Nielsen, M. A. \& Chuang, I. L. Quantum Computation and Quantum Information (Cambridge Univ. Press, 2000).

6. Bruß, D. \& Leuchs, G. Lectures on Quantum Information (Wiley-VCH, 2007)

7. Scully, O. M., Englert, B.-G. \& Walther, H. Quantum optical tests of complementarity. Nature 351, 111-116 (1991)

8. Schmidt, L. Ph. H. et al. Momentum transfer to a free floating double slit: realization of a thought experiment from the Einstein-Bohr debates. Phys. Rev. Lett. 111, 103201 (2013).

9. Marshall, W., Simon, C., Penrose, R. \& Bouwmeester, D. Towards quantum superpositions of a mirror. Phys. Rev. Lett. 91, 130401 (2003).
10. Arndt, M. \& Hornberger, K. Testing the limits of quantum mechanical superpositions. Nat. Phys. 10, 271-275 (2014).

11. Ionicioiu, R. \& Terno, D. R. Proposal for a quantum delayed-choice experiment. Phys. Rev. Lett. 107, 230406 (2011).

12. Céleri, L. et al. Quantum control in foundational experiments. Found. Phys. $\mathbf{4 4}$ 576-587 (2014).

13. Tang, J.-S., Li, Y.-L., Li, C.-F. \& Guo, G.-C. Revisiting Bohr's principle of complementarity using a quantum device. Phys. Rev. A 88, 014103 (2013).

14. Qureshi, T. Modified Two-Slit Experiments and Complementarity. Preprint at http://arxivorg/abs/1205.2207 (2012).

15. Bohr, N. in Albert Einstein-Philosopher-Scientist (ed. Schlipp, P. A.) 200-241 (The Library of Living Philosophers, 1949).

16. Wheeler, J. A. in Quantum Theory and Measurement (eds Wheeler, J. A. \& Zurek, W. H.) 182-213 (Princeton Univ. Press, 1984).

17. Leggett, A. J. in Compendium of Quantum Physics (eds Greenberger, D., Hentschel, K. \& Weinert, F.) 161-166 (Springer, 2009).

18. Jacques, V. et al. Experimental realization of Wheeler's delayed-choice Gedanken experiment. Science 315, 966-968 (2007).

19. Kaiser, F. et al. Entanglement-enabled delayed choice experiment. Science 338, 637-640 (2012).

20. Roy, S., Shukla, A. \& Mahesh, T. S. NMR implementation of a quantum delayed-choice experiment. Phys. Rev. A 85, 022109 (2012).

21. Auccaise, R. et al. Experimental analysis of the quantum complementarity principle. Phys. Rev. A 85, 032121 (2012).

22. Tang, J-S. et al. Realization of quantum Wheeler's delayed-choice experiment. Nat. Photonics 6, 600-604 (2012).

23. Peruzzo, A. et al. A quantum delayed choice experiment. Science 338, 634-637 (2012).

24. Langford, N. K. et al. Demonstration of a simple entangling optical gate and its use in Bell-state analysis. Phys. Rev. Lett. 95, 210504 (2005).

25. Kiesel, N. et al. Linear optics controlled-phase gate made simple. Phys. Rev Lett. 95, 210505 (2005).

26. Kaltenbaek, R. et al. Experimental Interference of Independent Photons. Phys. Rev. Lett. 96, 240502 (2006).

27. Kaltenbaek, R., Prevedel, R., Aspelmeyer, M. \& Zeilinger, A. High-fidelity entanglement swapping with fully independent sources. Phys. Rev. A 79, 040302(R) (2009).

28. Ionicioiu, R., Mann, R. B. \& Terno, D. R. Determinism, independence and objectivity are inconsistent. Preprint at http://arxivorg/abs/1406.3963 (2014).

29. Branciard, C., Gisin, N. \& Pironio, S. Characterizing the nonlocal correlations created via entanglement swapping. Phys. Rev. Lett. 104, 170401 (2010).

\section{Acknowledgements}

D.R.T. thanks Perimeter Institute for support and hospitality. We thank Lucas Céleri, Jim Cresser, Berge Englert, Peter Knight, Stojan Rebić, Valerio Scarani, Vlatko Vedral and Man-Hong Yung for discussions and critical comments and Alla Terno for help with visualization. This work was supported in part by the Natural Sciences and Engineering Research Council of Canada. R.I. acknowledges support from the Institute for Quantum Computing, University of Waterloo, Canada, where this work started.

\section{Author contributions}

T.J. and R.I. conceived the entanglement-controlled protocol. D.R.T. performed the hidden-variable analysis. R.I. and T.J. produced the experimental design. R.B.M. and D.R.T. analysed the experimental signatures. All authors contributed to the writing of the manuscript. D.R.T. coordinated the project.

\section{Additional information}

Competing financial interests: The authors declare no competing financial interests.

Reprints and permission information is available online at http://npg.nature.com/ reprintsandpermissions/

How to cite this article: Ionicioiu, R. et al. Is wave-particle objectivity compatible with determinism and locality? Nat. Commun. 5:4997 doi: 10.1038/ncomms5997 (2014). 


\section{Publisher Correction: Is wave-particle objectivity compatible with determinism and locality?}

Radu Ionicioiu, Thomas Jennewein, Robert B. Mann \& Daniel R. Terno

Nature Communications 5:4997 doi: 10.1038/ncomms5997 (2014); Published online 26 Sep 2014; Updated 26 Mar 2018

The original HTML version of this Article had an incorrect article number of 3997; it should have been 4997. This has now been corrected in the HTML; the PDF version of the Article was correct from the time of publication. 